\title{
Randomised Controlled Study to Evaluate the Outcomes between Rigid and Flexible Cystoscopy in Men with Lower Urinary Tract Symptoms Undergoing Diagnostic Cystoscopy
}

\author{
Gaurish Sawant ${ }^{1}$, Pawan Lal², Jagdeep Yadav ${ }^{3}$, Lovenish Kumar4, \\ Rahul Saini ${ }^{5}$, Tariq Hameed ${ }^{6}$, Haraesh Maranna ${ }^{7}$, Rahul Bhatia ${ }^{8}$ \\ 1, 2, 3, 4, 5, 6, 7, 8 Department of Surgery, Maulana Azad Medical College\& Associated Hospitals, New Delhi, India.
}

\section{ABSTRACT}

\section{BACKGROUND}

Cystoscopy is a commonly performed routine outpatient procedure and an essential diagnostic tool in urology. It may be performed using either rigid or flexible cystoscopes. From the clinical perspective, it might appear that flexible cystoscopy causes less discomfort than rigid cystoscopy, but remarkably head-to- head comparisons of these two devices are sparse. We wanted to compare rigid cystoscopy with flexible cystoscopy in men with lower urinary tract symptoms (LUTS), in terms of the post-procedure outcomes defined as discomfort / pain experienced by the patient using the visual analogue score (VAS) scoring and duration of the procedure.

\section{METHODS}

A total of 60 male patients with LUTS were randomised to flexible and rigid cystoscopy groups ( $\mathrm{N}=30$ each group). Cystoscopy was done using appropriate rigid and flexible cystoscope. Post procedure, patient was assessed with VAS scaling for pain. The duration of the procedure and any post-procedure discomfort and complication were also noted.

\section{RESULTS}

The mean age of patients of flexible cystoscopy group was comparable to that of a rigid cystoscopy group $(39.43 \pm 11.45$ vs. $41.7 \pm 10.8, \mathrm{P}=0.433)$. The mean duration (minutes) for flexible cystoscopy was significantly less than that of rigid cystoscopy $(8.53 \pm 1.81$ vs. $9.77 \pm 2.14, \mathrm{P}=0.019)$. The mean VAS score for flexible cystoscopy was significantly less than that of rigid cystoscopy $(2.57 \pm 0.97$ vs. $3.97 \pm 1.1, \mathrm{P}<$ 0.0001 ). There was no significant difference between groups in terms of diagnosis ( $P$ $=0.796$ ). Post-procedure complications were also comparable between the two groups.

\section{CONCLUSIONS}

Flexible cystoscopy caused less pain and discomfort as compared to rigid cystoscopy. Duration of procedure was also less for the flexible cystoscope.

\section{KEY WORDS}

Cystoscopy, Flexible Cystoscopy, Lower Urinary Tract Symptoms, Pain, Rigid Cystoscopy
Corresponding Author:

Dr. Gaurish Sawant,

\#90, Arjun Nagar, Safdarjung enclave

New Delhi - 110029, India.

E-mail: gaurish1311@outlook.com

DOI: $10.14260 / \mathrm{jemds} / 2021 / 191$

How to Cite This Article:

Sawant G, Lal P, Yadav J, et al. Randomised controlled study to evaluate the outcomes between rigid and flexible cystoscopy in men with lower urinary tract symptoms undergoing diagnostic cystoscopy. J Evolution Med Dent Sci 2021;10(12):888893, DOI: $10.14260 / \mathrm{jemds} / 2021 / 191$

Submission 28-09-2020,

Peer Review 24-01-2021,

Acceptance 02-02-2021,

Published 22-03-2021.

Copyright (C) 2021 Gaurish Sawant et al. This is an open access article distributed under Creative Commons Attribution License [Attribution 4.0 International (CC BY 4.0)] 


\section{BACKGROUND}

In the current times, cystoscopy has become a very important diagnostic tool for the management of urological cases which has allowed us to save time, to manage our resources in an efficient way and use the right treatment modality. It is an endoscopic method which is used to visualise the urethra and the bladder. Cystoscopy is a minimally invasive procedure that doesn't require a full-fledged operating theatre set up but can be done as outpatient-based procedure, which has many indications due to its versatility in urological management of the patient. It is the gold standard for bladder cancer detection and follow-up in superficial tumours and it is commonly used for evaluation of hematuria, voiding symptoms, and performance of minor procedures such as foreign body removal.1-3 Cystoscopy may be performed using either rigid or flexible cystoscopes. Rigid cystoscopes were the first instruments used, followed by flexible devices, which were introduced by Tsuchida and Sugawara in 1973.4

Compared with flexible cystoscopes, rigid instruments offer better image quality, a wider lumen of the working channel, improved irrigant flow and superior handiness. However, flexible cystoscopes provide more options for patient positioning, enable smooth passage over an elevated bladder neck or median lobe, facilitate full inspection of the bladder because of their movable tip and, what is most important is it significantly improves patient comfort (patient can be positioned in any comfortable position). ${ }^{5}$

The observations we do during diagnostic cystoscopy are influenced by a variety of factors. One of them is the vision offered by the cystoscope used for the procedure, and hence rigid cystoscopies with superior optics excel in this regard. Another very important factor is the patient comfort during the diagnostic procedure. As this is outpatient procedure carried out under the effect of local anaesthesia, many times, we tend to miss an observation due to patient being uncooperative. This mostly happens when the patient is uncomfortable and experiencing a certain degree of pain which is beyond the normal limit of tolerability of the patients.

The discomfort experienced by the patient can be due to the fact that the patient is positioned in an awkward way, like in lithotomy position for rigid cystoscopy. Another reason for discomfort is the pain experienced during the procedure, especially when negotiating the curved male urethra, which if exceeds the patients' threshold makes them compliant about the procedure leading to abandoning the procedure. Local factors such as underlying inflammation of the urinary tract, haziness of the media, hematuria, urinary stricture, etc. also interfere in the procedure of diagnostic cystoscopy preventing from having a full view of the entire system.

From the clinical perspective, it might be conclusive that flexible cystoscopy causes less discomfort than rigid cystoscopy, but remarkably head-to-head comparisons of these two devices are limited to a few studies, and they are based on heterogeneous groups of patients and / or disorders, and some of them have unclear methodology or include rigid cystoscopy in general anaesthesia, which is no longer routinely performed nowadays. ${ }^{4}$

The objective of this study was to perform a randomised study, comparing the outcomes of the rigid and flexible cystoscopy in terms of pain / discomfort experienced by the patient and the duration of the procedure.

\section{METHODS}

This randomised control study was conducted at the Department of General Surgery, Maulana Azad Medical College and associated Lok Nayak Hospital, New Delhi for a period of 18 months from May 2017 to Oct 2018. The study population included patients admitted from casualty and outpatient department. The study was conducted after taking approval from the institutional ethical board (Approval number, F. No. / 11 / IEC / MAMC / 2016 / 13).

Inclusion criteria included male patients 18 years and above of age with lower urinary tract symptoms (obstructive and irritative). Exclusion criteria included patients who were undergoing cystoscopies with interventions (e.g. stent removal, biopsies), who were with suprapubic catheter, who were with clinical evidence of urethral stricture and prostatitis, presence of the history of radical prostatectomy or cystoprostatectomy, and urine culture-positive patients.

The sample size calculation was based on the study of Islamoglu $\mathrm{E}$ et al. who observed that the mean visual analogue scale scores after the procedure were significantly lower for the flexible arm compared to the rigid arm $(2.57 \pm 1.83$ and $4.48 \pm 2.18$, respectively, $\mathrm{P}<0.01) \cdot{ }^{6}$ Taking these values as reference, the minimum required sample size with $95 \%$ power of study and $5 \%$ level of significance is 29 patients in each study group. To reduce margin of error, total sample size taken was 60 (30 patients per group).

The primary outcome was discomfort experienced immediately post procedure by the patient defined as a VAS score of $>3$. The secondary outcome was time taken in the procedure, which was calculated from the moment of introducing the cystoscope through the meatal opening in the penis till removing the scope totally out of the urethra visualising the urogenital system.

Informed consent was taken from all the patients before the enrolment. Those who fulfilled the inclusion criteria were enrolled. On the day of the procedure, assessment of the patient was done, and informed consent was taken, and the patient was explained about the procedure of VAS scoring. Parts were prepared with povidone iodine, after patient was positioned in lithotomy position for rigid cystoscopy and supine position for flexible cystoscopy. Urethra instilled with $10 \mathrm{ml}$ of $2 \%$ lignocaine, with dwell time for 5 minutes. $^{7}$ Cystoscopy was done using the appropriate rigid and flexible cystoscope. We used an Olympus CYF-5 flexible cystoscope and a Karl Storz 19F rigid cystoscope. Post procedural patient was assessed with visual analogue score scaling for pain.4,8,9 Also the duration of the procedure and any post-procedure discomfort and complication was taken into account.

\section{Statistical Analysis}

Categorical variables were presented in number and percentage (\%) and continuous variables were presented as mean \pm SD and median. Normality of data was tested by Kolmogorov-Smirnov test. If the normality was rejected, then nonparametric test was used. Statistical tests were applied as follows - 
- Quantitative variables were compared using Independent $\mathrm{t}$ test / Mann-Whitney test (when the data sets were not normally distributed) between the two groups.

- Qualitative variables were correlated using chi- square test.

- Spearman rank correlation coefficient was used to assess the correlation of duration with VAS score.

A P-value of $<0.05$ was considered statistically significant. The data was entered in MS Excel spreadsheet and analysis was done using Statistical Package for Social Sciences (SPSS) version 21.0.

\section{RESULTS}

Total 60 patients with LUTS who underwent diagnostic cystoscopy were included with 30 patients in each group of rigid and flexible cystoscopy.

The minimum and maximum age for flexible cystoscopy group was 22 years and 64 years, respectively. The minimum and maximum age for rigid cystoscopy group was 25 years and 61 years, respectively. The mean age of patients of flexible cystoscopy group was comparable to that of rigid cystoscopy group (39.43 \pm 11.45 vs $41.7 \pm 10.8, \mathrm{P}=0.433$ ) (Table 1 )

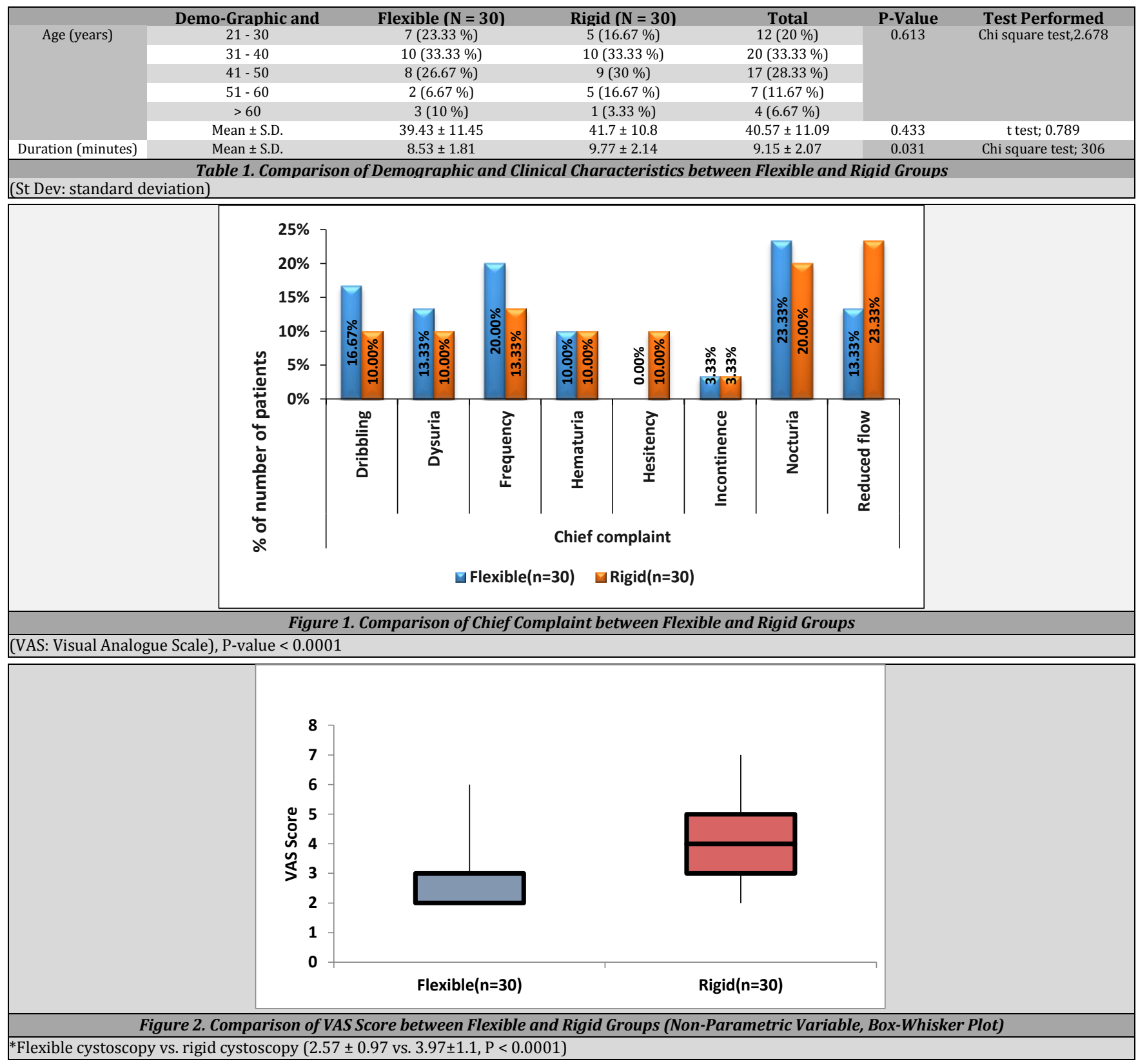

\section{Duration of Procedure}

The shortest and longest duration for flexible cystoscopy was 5 minutes and 12 minutes, respectively. The mean duration (minutes) for flexible cystoscopy was significantly less than that of rigid cystoscopy $(8.53 \pm 1.81$ VS $9.77 \pm 2.14, \mathrm{P}=0.019)$. Thus, flexible cystoscopy took lesser amount of time as compared to rigid cystoscopy. (Table 1)

\section{Chief Complaints}

The presenting chief complaints of lower urinary tract symptoms of the patients undergoing flexible cystoscopy were as follows: 7 out of 30 had nocturia, 6 had urinary frequency, 5 had dribbling of urine, 4 had dysuria, 4 had reduced flow of urinary stream, 3 of them had hematuria, and 1 patient had urinary incontinence. 
Similarly, in the rigid cystoscopy group, 7 out of 30 patients had reduced flow, while 6 had nocturia, 4 had increased urinary frequency, 3 had dribbling of urine, 3 of them had dysuria, 3 had hematuria, 3 had hesitancy in micturition, and 1 had incontinence. However, chief complaints were comparable in both groups $(\mathrm{P}=0.668)$.

Overall, the most common complaint of the patients was nocturia that was noted in 13 out of 60 patients $(21.6 \%)$ followed by reduced flow of urinary stream in 11 out of 60 patients (18.33\%), and increaser urinary frequency in 10 out of 60 patients $(16.66 \%$ ) (Figure 1$)$.

\begin{tabular}{|c|c|c|c|c|c|}
\hline Diagnosis & $\begin{array}{l}\text { Flexible } \\
(N=30)\end{array}$ & $\begin{array}{c}\text { Rigid } \\
(N=30)\end{array}$ & Total & P-Value & $\begin{array}{c}\text { Test } \\
\text { Performed }\end{array}$ \\
\hline BPH & $15(50 \%)$ & $14(46.67 \%)$ & $29(48.33 \%)$ & \multirow{3}{*}{0.796} & \multirow{3}{*}{$\begin{array}{l}\text { Chi square } \\
\text { test, } 0.067\end{array}$} \\
\hline UTI & $15(50 \%)$ & $16(53.33 \%)$ & $31(51.67 \%)$ & & \\
\hline Total & $30(100 \%)$ & $30(100 \%)$ & $60(100 \%)$ & & \\
\hline \multicolumn{6}{|c|}{ Table 2. Comparison of Diagnosis between Flexible and Rigid Groups } \\
\hline \multicolumn{6}{|c|}{ (BPH: benign prostatic hyperplasia; UTI: urinary tract infection) } \\
\hline \multirow{2}{*}{\multicolumn{3}{|c|}{ Variables }} & & \multicolumn{2}{|c|}{ VAS Score } \\
\hline & & & & Flexible & Rigid \\
\hline \multicolumn{6}{|c|}{ Duration (minutes) } \\
\hline \multicolumn{4}{|c|}{ Correlation coefficient } & 0.246 & 0.231 \\
\hline \multicolumn{4}{|c|}{ P value } & 0.190 & 0.219 \\
\hline \multicolumn{6}{|c|}{ Table 3. Correlation of Duration with VAS Score } \\
\hline \multicolumn{6}{|c|}{ Spearman rank correlation coefficient (VAS: visual analogue scale) } \\
\hline
\end{tabular}

\section{Cystoscopy Findings and Diagnosis}

In bladder, back pressure changes were seen in 7 (23.33\%) patients who underwent flexible cystoscopy and in 9 (30\%) patients who underwent rigid cystoscopy. There was no statistically significant difference in back pressure changes in patients who underwent flexible cystoscopy and rigid cystoscopy (P-value $=0.559$ ).

Bilateral UV openings were found to be normal in all the patients who underwent rigid and flexible cystoscopy. $(\mathrm{P}=1)$ Enlarged prostate was found in 15 (50\%) patients who underwent flexible cystoscopy and $14(46.67 \%)$ patients of rigid cystoscopy, which was comparable $(\mathrm{P}$-value $=0.796$ ).

Urethra was found to be normal in all the patients who underwent rigid and also flexible cystoscopy. $(\mathrm{P}=1)$

Out of the 30 patients who underwent diagnostic flexible cystoscopy, 15 patients were diagnosed with benign prostatic hyperplasia (BPH), and the rest 15 were diagnosed as urinary tract infections (UTI). Whereas, in the patients who underwent diagnostic rigid cystoscopy, 14 patients were diagnosed with BPH and the rest 16 were diagnosed with UTI. Overall, 31 (51.67 \%) patients had UTI while 29 (48.33\%) patients had $\mathrm{BPH}$. There was no significant difference between groups in terms of diagnosis (P-value $=0.796)$. Hence, flexible cystoscope is as good as rigid cystoscope for diagnostic cystoscopy. Also, to make a note regarding post-procedure complications, we evaluated the post-procedure urine culture analysis of all our patients. Only 2 patients in rigid group and 1 patient in the flexible group had a mixture of growth in the culture, which is considered to be non-significant, none of our patients had any sort of urethral injuries or dysuria after the procedure (Table 2).

\section{VAS Score and Discomfort Experienced}

The minimum VAS score for flexible cystoscopy was 2 while the maximum score was 6 . The minimum VAS score for rigid cystoscopy was 2 while the maximum was 7. The mean VAS score for flexible cystoscopy was significantly less than that of rigid cystoscopy $(2.57 \pm 0.97$ vs $3.97 \pm 1.1$, $\mathrm{P}<0.0001)$. Also, as per our definition of discomfort, 25 out of the 30 patients $(83.33 \%)$ undergoing flexible cystoscopy felt no discomfort and only 11 out of 30 patients $(16.67 \%)$ undergoing rigid cystoscopy felt no discomfort, with a P-value of 0.001 which is statistically significant. Thus, patients undergoing flexible cystoscopy felt less pain and discomfort as compared to rigid cystoscopy (Figure 2). Spearman rank correlation showed a positive correlation of duration of the procedure with VAS score, however, statistically it was not significant. $(\mathrm{P}>0.05)$ (Table 3)

\section{DISCUSSION}

In the present study, we have evaluated the outcomes of diagnostic cystoscopy comparing rigid and flexible cystoscopes on the basis of patient discomfort and procedure duration. This helps in knowing which instrument-rigid or flexible-has better patient acceptance without compromising on the diagnostic accuracy.

\section{Age Distribution}

In our study, the mean age of the flexible cystoscopy group was $39.43 \pm 11.45$ and that of rigid cystoscopy group was $41.7 \pm$ 10.8. Both the groups were comparable (P-value was 0.433 ) the youngest patient was 22 years old and the oldest was 64 years. In a study conducted in 2005 by Chen YT et al. the mean age was $63.0 \pm 15.3$ years. ${ }^{10}$ Likewise in a study by Seklehner S et al. the mean age was 64.9 years and 67.6 years respectively for patients undergoing rigid and flexible cystoscopy. ${ }^{4} \mathrm{~A}$ study by Casteleijn et al. was conducted on female patients with indications for which a cystoscopy was performed including hematuria, LUTS, and recurrent urinary tract infections; mean age of the patients was $58 \pm 17$ years. ${ }^{11}$ Üçer 0 et al. reported that mean age was comparable in both flexible cystoscopy and rigid cystoscopy groups. ${ }^{12}$

\section{Duration of Procedure}

During the diagnostic cystoscopy we conducted on the 60 patients in our study, we measured the mean duration of procedure for flexible cystoscopy was $8.53 \pm 1.81$ minutes; whereas, that for the rigid cystoscopy was $9.77 \pm 2.14$ minutes. Thus, duration of the procedure for flexible cystoscopy was significantly lower than that of rigid cystoscopy $(\mathrm{P}=0.019)$. As diagnostic cystoscopy is conducted on an outpatient basis and under local anaesthesia, the longer the procedure takes the more discomfort the patient experience. Also, the patient undergoing rigid cystoscopy under local anaesthesia is kept in a lithotomy position and so has to bear with the uncomfortable position for a longer time. For the examiner, the rigid scope has to be negotiated and maneuvered in all possible directions so as to get the full picture of the bladder as compared to the flexible cystoscopy which is conducted in a supine position. In flexible cystoscopy, the patient is at much more ease and hence, is more cooperative and the flexibility of the tip of the cystoscope helps in proper visualisation of the entire bladder. 
In a study conducted by Tepeler A et al. it was observed that the procedure time for rigid cystoscopy was far prolonged that that for flexible cystoscopy $(19.7 \pm 2.9$ minutes $\mathrm{v} / \mathrm{s} 9.92$ \pm 2.3 minutes $).{ }^{13}$

\section{Chief Complaints and Diagnosis}

In our study of the 60 patients who presented to us with the chief complaint of LUTS, we found that the patients had a variety of symptoms, most common of which were nocturia, reduced flow of the urinary stream, increased frequency of micturition, dribbling of urine, urinary hesitancy and incontinence, and also hematuria. We observed that there was a mix of both obstructive and irritative symptoms.

We also observed that among the patients who underwent diagnostic cystoscopy, UTI was present in 31 patients and BPH in 29 patients. We were able to note all the diagnostic findings excellently during the procedure, As is the general consensus that rigid cystoscopy provides a better vision than the flexible counterpart, we in our study found that the flexible cystoscope was as good as the rigid cystoscope while noting the cystoscopy findings and causing much less discomfort to the patient.

Also, to make a note that in our study we did not find any significant post-procedure complications such as bacteruria, pyuria, hematuria, or injury of any sorts, implying that if conducted in a proper technique and with experienced hands most of the complications can be avoided. As demonstrated by Almallah YZ et al. in his study the risk of bacteruria in flexible cystoscopy is low and is therefore a safe procedure, and do not require any antibiotic prophylaxis. ${ }^{14}$ Similar findings were reported by Burke DM et al. who reported a $2.7 \%$ risk of developing a urinary infection after flexible cystoscopy. ${ }^{15}$ Turan et al. reported that after rigid cystoscopy, $8 \%$ patients developed significant bacteriuria, and $8 \%$ patients developed pyuria without significant bacteriuria. Bacteraemia was not determined in any of the patients. ${ }^{16}$

\section{VAS Score and Discomfort Experienced}

In our study out of the 60 patients, 30 patients had flexible cystoscopy done, and rest 30 had rigid cystoscopy. The mean score for flexible cystoscopy was significantly less than that of rigid cystoscopy $(2.57 \pm 0.97$ vs. $3.97 \pm 1.1, \mathrm{P}=0.001)$. As there was a significant difference in the pain and discomfort experienced by the patients, this shows that flexible cystoscopy has a better outcome and provides more comfort to the patient improving compliance with the procedure. The flexible cystoscope has a smaller caliber and due to the deflection at the tip of the scope it is better able to negotiate the bend at the junction of membranous and prostatic urethra causing less stretching and hence less pain; also for the entire duration of the procedure, the patient is in a supine position which is more comfortable than lithotomy position especially for the older population in whom LUTS is more common.

The rigid cystoscope has a larger caliber and hence causes more stretching of the urethral mucosa, especially when negotiating the curved male urethra, which causes more discomfort. Other factors that influence the pain perception during cystoscopy are the utilisation of lubricants and examiners experience, especially with rigid cystoscopy.
In a study by Seklehner S et al. mean pain level were low in flexible cystoscopy as compared to rigid cystoscopy as indicated by the VAS score (flexible vs rigid, 1.1 vs 2.3 ). ${ }^{4}$ Also noted was that more men were pain free or experienced mild pain during flexible cystoscopy as compared to rigid cystoscopy (89.3\% in flexible cystoscopy). They concluded that their group of patients were more compliant and experienced less discomfort with flexible cystoscopy. Similarly, in a study conducted by Denholm, it was observed that flexible cystoscopy was more painless than rigid cystoscopy. ${ }^{17}$

In another study conducted by Krajewski W et al. pain was compared in men undergoing cyclical cystoscopic assessment with rigid and flexible cystoscopy after transurethral resection of bladder tumour. It was noted that flexible cystoscopy patients reported lower pain as compared to rigid counterpart (mean VAS score $2.1 \mathrm{v} / \mathrm{s}$ 5.7). ${ }^{5}$ In a similar study by Islamoglu et al. the mean VAS scores after the procedure were significantly lower for the flexible arm compared to the rigid $\operatorname{arm}(2.57 \pm 1.83$ vs. $4.48 \pm 2.18, \mathrm{P}<0.01)$. Patients undergoing flexible cystoscopy experienced less pain then they expected in contrast to the patients in the rigid arm $(\mathrm{P}<0.01){ }^{6}$ Casteleijn et al. reported that the median VAS score was significantly lower for women undergoing flexible cystoscopy compared to rigid cystoscopy ( 0 vs. $15, \mathrm{P}<0.001) .11$

Chen YT et al. reported a VAS score of $2.8 \pm 1.1$ in his study of flexible cystoscopy in patients using $2 \%$ lignocaine ointment. ${ }^{10}$

In contrast to our findings, no significant difference in VPS score was found by Üçer 0 et al. in both groups. ${ }^{12}$

The patients sometimes experience discomfort beyond their level of co-operation, and hence the procedure has to abandoned. In these cases, the patient is worked up and is made fit to undergo the cystoscopy procedure under general anaesthesia. In men, flexible cystoscopy is better tolerated as compared to rigid cystoscopy, which may be because of the urethral length and the angle needed for inspecting the bladder.

The small sample size is one of the limitations of this study. Another limitation was that it was a single-center study, so results cannot be generalized. So, a larger multicentric trial is recommended for the confirmation of these findings. Thirdly, we had not compared the cost efficiency of both techniques as well as the discomfort faced by the operating surgeon. Lastly, long-term follow up of the patients was not done, thus it is difficult to assess the late and long-term complications of diagnostic rigid cystoscopy.

\section{CONCLUSIONS}

Patients who underwent flexible cystoscopy had a significantly lower VAS score than those who had undergone rigid cystoscopy. Flexible cystoscopy caused less discomfort to patients than rigid cystoscopy. Thus, flexible cystoscopy caused less pain and discomfort in patients. Duration of the procedure was significantly less for flexible cystoscopy as compared to rigid cystoscopy.

Both flexible and rigid cystoscopes are equally good in patients with LUTS in establishing the diagnosis with both providing comparable vision and observations. 
To conclude, flexible cystoscopy has a better outcome than rigid cystoscopy in men with LUTS undergoing diagnostic cystoscopy. It is a faster procedure which causes less discomfort.

Data sharing statement provided by the authors is available with the full text of this article at jemds.com.

Financial or other competing interests: None.

Disclosure forms provided by the authors are available with the full text of this article at jemds.com.

\section{REFERENCES}

[1] Amling CL. Diagnosis and management of superficial bladder cancer. Curr Probl Cancer 2001;25(4):219-78.

[2] Gee JR, Waterman BJ, Jarrard DF, et al. Flexible and rigid cystoscopy in women. JSLS 2009;13(2):135-8.

[3] Rafique M. Intravesical foreign bodies: review and current management strategies. Urol J 2008;5(4):223-31.

[4] Seklehner S, Remzi M, Fajkovic H, et al. Prospective multiinstitutional study analyzing pain perception of flexible and rigid cystoscopy in men. Urology 2015;85(4):737-41.

[5] Krajewski W, Zdrojowy R, Wojciechowska J, et al. Patient comfort during flexible and rigid cystourethroscopy. Wideochir Inne Tech Maloinwazyjne 2016;11(2):94-7.

[6] Islamoglu E, Akdoğan B, Yüksel S, et al. The effect of flexible and rigid cystoscopy on quality of life, pain perception and lower urinary tract symptoms: a prospective randomized study. Akdeniz Med J 2018;3:228-32.

[7] Patel AR, Jones JS, Babineau D. Lidocaine $2 \%$ gel versus plain lubricating gel for pain reduction during flexible cystoscopy: a meta - analysis of prospective, randomized, controlled trials. J Urol 2008;179(3):986-90.

[8] Soomro KQ, Nasir AR, Ather MH. Impact of patient's selfviewing of flexible cystoscopy on pain using a visual analog scale in a randomized controlled trial. Urology 2011;77(1):21-3.

[9] Gould D, Kelly D, Goldstone L, et al. Examining the validity of pressure ulcer risk assessment scales: developing and using illustrated patient simulations to collect the data. J Clin Nurs 2001;10(5):697-706.

[10] Chen YT, Hsiao PJ, Wong WY, et al. Randomized doubleblind comparison of lidocaine gel and plain lubricating gel in relieving pain during flexible cystoscopy. J Endourol 2005;19(2):163-6.

[11] Casteleijn NF, Vriesema JL, Stomps SP, et al. The effect of office based flexible and rigid cystoscopy on pain experience in female patients. Investig Clin Urol 2017;58(1):48-53.

[12] Üçer O, Temeltaş G, Yüksel MB, et al. Comparison of pain, quality of life, lower urinary tract symptoms and sexual function between flexible and rigid cystoscopy in followup male patients with non muscle invasive bladder cancer: a randomized controlled cross section single blind study. Eur Urol 2017;16(Suppl 3):e1159.

[13] Tepeler A, Silay MS, Akman T, et al. Comparison of flexible and rigid cystoscopy - assisted ureteral catheter insertion before percutaneous nephrolithotomy: a prospective randomized trial. J Endourol 2013;27(6):722-6.

[14] Almallah YZ, Rennie CD, Stone J, et al. Urinary tract infection and patient satisfaction after flexible cystoscopy and urodynamic evaluation. Urology 2000;56(1):37-9.

[15] Burke DM, Shackley DC, O'Reilly PH. The communitybased morbidity of flexible cystoscopy. BJU Int 2002;89(4):347-9.

[16] Turan H, Balci U, Erdinc FS, et al. Bacteriuria, pyuria and bacteremia frequency following outpatient cystoscopy. Int J Urol 2006;13(1):25-8.

[17] Denholm SW, Conn IG, Newsam JE, et al. Morbidity following cystoscopy: comparison of flexible and rigid techniques. Br J Urol 1990;66(2):152-4. 